\title{
TAXONOMIA SETORIAL COM INDICADORES DE ESFORÇO INOVATIVO*
}

\author{
Evaldo Henrique da Silva
}

RESUMO: O propósito deste trabalho é a construção de uma taxonomia do setor manufatureiro da indústria brasileira, a qual utiliza como critério de classificação as variáveis indicadoras do esforço inovativo das firmas. Essa escolha é devida a dois pressupostos. O primeiro é que o desempenho competitivo e econômico-financeiro no âmbito das indústrias está associado ao desempenho inovativo das firmas que as constituem. O segundo pressuposto é de que esse esforço obedece a imperativos ou regimes tecnológicos que são específicos de cada indústria ou setor. Foi utilizada exclusivamente a análise de cluster como método de agrupamento. A ênfase nas variáveis consideradas inputs da inovação e o uso exclusivo da análise de cluster são particularidades dessa taxonomia. Os resultados indicam que a indústria brasileira é dominada por quatro grupos setoriais característicos. Contudo, as diferenças entre esses grupos são sutis, pois entre as variáveis analisadas são poucas as que fizeram a diferença entre os grupos, e essa diferença não apresenta desníveis profundos. Suspeita-se da tendência de predomínio de setores considerados como de baixa intensidade tecnológica na indústria brasileira.

PALAVRAS-CHAVE: Taxonomia industrial; competitividade; inovação.

CLASSIFICAÇÃO JEL: O33.

\footnotetext{
* Artigo recebido em 16/04/2012 e aprovado em 05/11/2012.

a Doutor em Economia pela Universidade Federal do Rio de Janeiro (UFRJ) e professor do Departamento de Economia da Universidade Federal de Viçosa (UFV). Contato: ehsilva@ufv.br.
} 


\section{SECTORAL TAXONOMY WITH INDICATORS OF INNOVATIVE EFFORT}

ABSTRACT: The purpose of this study is to build a taxonomy of the Brazilian manufacturing industry based on the innovative efforts of the companies. This choice is due to two assumptions. First, competitiveness and financial-economic performance in a given industry is related to the innovative performance of its firms. Second, the innovative effort meets the requirements or technological regimes that are specific to each industry or sector. The use of cluster analyses as the only method of grouping and the emphasis on indicators of innovation inputs are peculiarities of this taxonomy. The results indicate that the Brazilian industry is dominated by four typical groups of sectors - with no deep, significant differences among them - and a predominance of industries with low technological intensity.

KEYWORDS: Industrial taxonomy; competitiveness; innovation. 


\section{INTRODUÇÃO}

A construção de taxonomias industriais com ênfase nos indicadores de inovação é bastante comum no campo da economia neoschumpeteriana da inovação.

Essa ênfase é devida ao pressuposto de que os indicadores de competitividade e de desempenho econômico-financeiro das firmas ou das indústrias estão diretamente relacionados aos indicadores de inovação. Por outro lado, de acordo com a visão neoschumpeteriana, o impacto dos indicadores de inovação depende das especificidades tecnológicas de cada indústria, o que reforça o motivo da busca de taxonomias focadas nesses indicadores.

A taxonomia proposta neste artigo é coerente com essa linha de pesquisa, mas diferente de outras taxonomias da mesma linha nos seguintes aspectos: utiliza exclusivamente a análise de cluster e variáveis indicadoras de esforço de aprendizagem tecnológica no âmbito das firmas. Essas variáveis cumprem a função de inputs da inovação. A maior parte das taxonomias que empregam métodos consagrados de agrupamento, como a análise fatorial e de cluster, agrega em um único modelo variáveis indicadoras do esforço e do desempenho inovativo, isto é, variáveis de inputs e outputs da inovação.

A opção metodológica deste artigo tem por base o princípio de que a combinação de inputs da inovação é condicionada por imperativos de natureza tecnológica, os quais são específicos para cada setor ou grupo setorial. Esses condicionantes são exaustivamente analisados no campo da teoria neoschumpeteriana da inovação, a partir dos conceitos de paradigma tecnológico e de regime tecnológico.

Os resultados da análise de cluster indicam que, no caso da economia brasileira, a indústria é dominada por quatro grupos setoriais característicos. Não obstante, as fontes de diferenciação desses grupos são relativamente sutis, pois abrangem um número relativamente pequeno do total de variáveis usadas na análise e não apresentam desníveis muito profundos.

Essa constatação levanta a questão a respeito de haver ou não uma tendência de homogeneização dos padrões setoriais de inovação na indústria brasileira. Pelos indicadores analisados, essa transformação está indo em direção à predominância de setores menos intensivos em termos de esforço inovativo, sobressaindo apenas os investimentos em novas máquinas e equipamentos, o que é típico do esforço inovativo em setores considerados de baixa intensidade tecnológica. 


\section{REGIMES TECNOLÓGICOS E PADRÕES SETORIAIS DE INOVAÇÃO}

Segundo Dosi (1988), a atividade inovativa representa o esforço de criação de algo novo a partir de uma base de conhecimento previamente acumulada. Em alguns setores, essa base é fortemente dependente do processo de aprendizagem por tentativa e erros, que se desenrola dentro da firma (learning by doing). Em outros setores, o maior peso advém dos conhecimentos gerados pelas pesquisas científicas, valendo a expressão learning from science and technology (Malerba, 1992). Existem outras variantes, a exemplo dos conhecimentos incorporados nos bens de capital e dos conhecimentos adquiridos via spillover, havendo também a possibilidade de combinações entre essas características. Em síntese, a natureza da base de conhecimento apresenta especificidades de âmbito setorial, as quais vão estabelecer o regime de aprendizagem tecnológica que impera em cada setor, moldando a atividade inovativa no âmbito das firmas.

A relação entre as especificidades da base de conhecimento e a dinâmica tecnológica e competitiva foi analisada pioneiramente por Nelson e Winter (1982), Winter (1984) e Pavitt (1984). Nos artigos de Cohen e Levinthal (1989) e de Malerba e Orsenigo (1993, 1997) são encontradas análises teóricas exaustivas visando demonstrar a versatilidade teórica e empírica do conceito de regime tecnológico para o entendimento da dinâmica industrial e tecnológica.

Com a consolidação do conceito de regime tecnológico no campo da economia da inovação, surgem propostas de taxonomia industrial baseadas em indicadores tecnológicos. As mais conhecidas são as de OCDE (2007), a UNCTAD (2002) e de Lall (2000). No caso da taxonomia proposta pela OCDE é utilizada como critério de agrupamento a taxa de investimento em pesquisa e desenvolvimento (P\&D), exclusivamente. Nas taxonomias sugeridas pela UNCTAD e por Lall são utilizadas informações fornecidas por experts em tecnologias industriais - incluindo o próprio autor, no caso da taxonomia de Lall - as quais, por sua vez, foram empregadas para aprimorar a taxonomia de Pavitt (1984), produzindo, assim, as referidas taxonomias.

Uma nova linha de pesquisa foi inaugurada com o trabalho de Marsili (1999), Marsili e Verspagen (2001). Sob a influência direta das contribuições de Nelson e Winter (1982) e demais autores citados anteriormente, esses autores propõem a construção de uma taxonomia mais robusta, ligando os aspectos teóricos do conceito de regime tecnológico, as informações geradas por experts em tecnologias industriais, incluindo os próprios autores, e métodos estatísticos mais refinados, a exemplo da análise de cluster. Concomitantemente, os autores empregam métodos multivariados (análise fatorial, análise de componentes principais e análise discriminante) para testar a robustez da taxonomia sugerida. 
Para o caso brasileiro, uma contribuição relevante foi produzida por Campos e Urraca Ruiz (2009). A taxonomia proposta por esses autores resulta de um esforço de integração da taxonomia de Pavitt (1984) com métodos estatísticos mais refinados, particularmente a análise de cluster. Nesse caso, não foram empreendidos testes de robustez.

Fazendo uma comparação entre essas taxonomias nota-se que uma parte dos setores forma grupos similares em todas essas taxonomias, conquanto a posição taxonômica de uma parte dos setores varie de uma taxonomia para outra -, o que não é surpreendente, pois os critérios e/ou os métodos de agrupamento são diferentes. O fato importante é que todas essas taxonomias foram construídas tendo como fundamento a noção de regime de aprendizagem tecnológica, ainda que de forma muito superficial, a exemplo da taxonomia proposta pela OCDE.

A ocorrência de discrepâncias pode suscitar dúvidas sobre os padrões de mudança tecnológica e de concorrência prevalecentes nos setores para os quais não existe consenso a respeito de suas posições taxonômicas, o que cria uma lacuna para os estudos empíricos da dinâmica tecnológica ou mesmo uma falta de consenso sobre políticas tecnológicas no âmbito setorial.

Em tese, essas discrepâncias constituem algo natural, visto que o conceito de regime tecnológico não está absolutamente delimitado, sendo inclusive interpretado como um constructo por alguns autores, a exemplo de Guidolin e Martinelli (2008). Apesar disso, muitos autores estão convencidos de que as delimitações do conceito de regime tecnológico são muito claras, sendo elas: o grau de apropriabilidade; o grau de complexidade; o grau de cumulatividade; e o grau de oportunidades tecnológicas. Portanto, as limitações não estão no próprio conceito, mas sim nas dificuldades de medição desses graus. Desse modo, na ausência de dados estatísticos que possam medir diretamente esses graus, convém empregar como critérios de agrupamento somente as variáveis que podem ser consideradas como proxies dessas medidas.

A definição dessas proxies não é, obviamente, de fácil consenso. Porém, não é propósito deste artigo analisar as distorções taxonômicas derivadas dessa limitação dos dados. O propósito é somente desenvolver uma análise taxonômica que seja o mais condizente possível com o conceito de regime tecnológico dentro da realidade brasileira, o que implica a utilização das pesquisas nacionais de inovação (notadamente a Pesquisa Industrial de Inovação Tecnológica [PINTEC], produzida pelo Instituto Brasileiro de Geografia e Estatística [IBGE]), pois essas pesquisas são os únicos surveys de inovação disponíveis no Brasil. O uso dessas pesquisas impõe limites à análise proposta, pois elas não operam diretamente com o conceito de regime tecnológico. Nesse caso, fez-se uma avaliação cuidadosa das variáveis que de algum modo podem refletir as características 
do regime tecnológico. Essas variáveis trazem informações sobre as fontes e os modos com que as firmas obtêm os conhecimentos que geram inovações.

Outro aspecto fundamental da construção de taxonomias reside nas complexas metodologias de validação dessas taxonomias. Na verdade, entre as taxonomias citadas somente a taxonomia proposta por Marsili e Verspagen (2001) foi submetida a testes exaustivos de robustez. A taxonomia proposta por Pavitt (1984) nasceu de uma base de dados muito rica a respeito de alguns detalhes importantes da atividade inovativa, mas não explorou variáveis diretamente associadas ao conceito de regime tecnológico. A taxonomia proposta pela OCDE é mais simples (em termos de sua construção) e mais difundida do que as outras. Não foi submetida diretamente a testes de robustez, mas o sucesso em termos de sua propagação é sintoma de que ela produziu agrupamentos relevantes, indicando que o grau de utilidade da taxonomia não está diretamente ligado ao grau de complexidade das metodologias empregadas na sua construção.

Nessa pesquisa não foi empreendida análise das metodologias empregadas na construção e no teste dessas taxonomias. A estratégia metodológica abarca o uso de um método objetivo de agrupamento: a análise de cluster, com seleção de variáveis (dentro das possibilidades da PINTEC) focadas no conceito de regime tecnológico. A avaliação da taxonomia ficou limitada aos critérios de seleção dos agrupamentos inerentes a análise de cluster e a uma comparação dos resultados a outras taxonomias. O uso exclusivo da análise de cluster, com variáveis extraídas da PINTEC, focalizadas no conceito de regime tecnológico e comparações a outras taxonomias, é um caminho metodológico inédito.

\section{METODOLOGIA}

A metodologia deste trabalho foi pautada no princípio da coerência para com o conceito de regime tecnológico proposto por Malerba e Orsenigo (1997). O primeiro ponto foi a escolha do conjunto de variáveis que podem espelhar da melhor forma possível as características do regime tecnológico. O segundo aspecto é uso exclusivo da técnica de cluster na formação do grupo, evitando assim critérios subjetivos e preservando a originalidade dos dados no processo de agrupamento.

A base de dados é as edições da PINTEC de 2003 e 2005. Nessa base não existem dados que possam medir diretamente as características do regime tecnológico. Não obstante, existe um conjunto de variáveis que caracterizam o modo com que as firmas desenvolvem o seu esforço de aprendizado. Foram essas as variáveis que integraram a análise de agrupamento (cluster analysis).

Diversas outras variáveis, a exemplo dos indicadores de desempenho tecnológico e econômico, as quais costumam integrar a análise de cluster de outros autores, a exemplo 
de Campos e Urraca Ruiz (2009) e de Guidolim e Martinelli (2008), não foram usadas pela razão óbvia de que essas variáveis não captam as características intrísecas do regime tecnológico nos moldes definidos por Malerba e Orsenigo (1997): os graus de oportunidade tecnológica; apropriabiliddade; cumulatividade; e complexidade. Porém, com certeza, essas variáveis podem captar os efeitos dessas características. O problema é a não existência de relação unívoca entre essas características e as variáveis indicadoras de desempenho. De acordo com Cohen e Levinthal (1989), setores que diferem significativamente em termos do grau de oportunidade tecnológica podem produzir valores relativamente baixos em termos de desempenho tecnológico, dada a influência das demais características.

O uso exclusivo da análise de cluster, o que é inédito em termos da literatura citada neste artigo, foi motivado pelo receio de se utilizarem critérios subjetivos, a exemplo de opiniões de experts em tecnologia industrial, o que, por sinal, está fora do alcance dos autores deste artigo, e também pelo reconhecimento de que não é prudente produzir agrupamentos unindo a técnica de cluster a outras técnicas que não foram projetadas para esse fim. O uso dessas outras técnicas é conveniente para o teste de robustez do agrupamento, quer dizer, elas são relevantes somente após à construção da taxonomia.

Alguns autores empregam a análise fatorial, a exemplo de Guidolin e Martinelli (2009), cujos resultados são utilizados na análise de cluster. Esse procedimento pode gerar resultados distorcidos, visto que esses métodos possuem lógicas absolutamente diferentes: 0 análise de cluster segue a lógica matemática na produção dos clusters, enquanto que a análise fatorial obedece à lógica estatística na identificação dos fatores.

$\mathrm{Na}$ análise fatorial são investigadas as correlações entre as variáveis, sendo a unidade de análise o elemento que garante a repetição do experimento, o que requer a hipótese que a fonte de dissimilaridade (variações) entre essas unidades são produzidas exclusivamente por fatores randômicos. $\mathrm{Na}$ analise de cluster, o foco recai justamente sobre a unidade de análise, tendo em vista a hipótese de que essas unidades estão distribuídas em grupos não homogêneos, que se distinguem em suas propriedades, características e qualidades.

A unidade de análise desta pesquisa é o setor, classificado pela CNAE em dois dígitos, pois a fonte de dados são as planilhas da PINTEC para os anos 2003 e 2005, disponibilizadas no site do IBGE. Os valores originais foram convertidos em valores percentuais, conforme indicado no anexo. Por sua vez, calculou-se a média simples desses valores entre 2003 e 2005. Essa conversão em valores percentuais permite que a análise de cluster possa ser feita sem a necessidade de padronização dos dados, o que possibilita utilizar a variabilidade entre as variáveis como um fator importante para determinar os clusters. 
As variáveis foram separadas em cinco grupos, conforme indicado no anexo. O primeiro grupo (conjunto $\mathrm{A}$ ) envolve variáveis que medem a opinião das empresas quanto à importância dada à determinada atividade inovativa implementada. Essas atividades podem ser definidas como "as etapas científicas, tecnológicas, organizacionais, financeiras e comerciais, incluindo o investimento em conhecimentos, que de fato conduzem, ou pretendem conduzir à implementação de inovações” (Manual de Oslo/OCDE, 2004, p. 104). Isso foi mensurado através da razão entre o número de empresas que implementaram inovação, atribuindo alta importância à determinada atividade inovativa, e o total dessas empresas.

O segundo grupo (conjunto B) mede diretamente os esforços de inovação das empresas. A mensuração adotada aqui coincide com a utilizada por Campos e Urraca Ruiz (2009), porém nesse último trabalho denominou-se o grupo de variáveis como "fontes de inovação". O terceiro grupo (conjunto C) de variáveis especifica as fontes de informação adotadas pelas empresas, sejam elas fontes abertas, como feiras e exposições, ou conhecimentos externos, sem cooperação ativa entre os agentes, podendo-se observar quais fontes foram consideradas pelas empresas como mais importantes para seu processo de aprendizado (Manual de Oslo/OCDE, 2004; Campos e Urraca Ruiz, 2009). Essas relações foram obtidas por meio do perfil dos trabalhadores ocupados nas atividades de P\&D (primeira parte) e também pela razão entre o número de empresas que implementaram inovações, atribuindo alta importância ao emprego de determinada fonte de informação, e o total de empresas que implementaram essas inovações (segunda parte).

No quarto grupo de variáveis (conjunto D) são observadas as formas de cooperação utilizadas pelas empresas que implementaram inovações. A relação de cooperação ocorre quando dois ou mais agentes operam de forma conjunta nos projetos de inovação, ou seja, há uma colaboração ativa, diferenciando-se das outras fontes de informação (Manual de Oslo/OCDE, 2004). Essas formas de cooperação foram medidas pela razão entre o número de empresas implementadoras de inovação, que atribuíram alta importância a determinada cooperação com outras organizações, e o total das empresas do setor que implementaram inovações.

Finalmente, o último grupo (conjunto E) serve para destacar o caráter da atividade de P\&D desenvolvida pelos setores e também como são alocados os trabalhadores nessas atividades.

Após a seleção das variáveis, determinou-se a matriz de dissimilaridade entre os setores por meio da distância euclidiana simples. Posteriormente, esses foram agrupados pelo método hierárquico de Ward. Através da observação do dendrograma, do pseudo-F e do $\mathrm{R}^{2}$, os setores foram agrupados em seis grupos distintos, permitindo obter os vetores de média de cada grupo, utilizando-os como sementes de agrupamento para o método 
não hierárquico, o que, por sua vez, possibilita uma realocação dos elementos entre os grupos. Um exemplo da união entre os métodos hierárquicos e os não hierárquicos pode ser encontrado em Campos e Urraca Ruiz (2009).

A escolha da distância euclidiana simples como medida de dissimilaridade deveu-se à utilização de variáveis métricas; ao comparar os resultados obtidos por essa medida com os obtidos pela distância euclidiana quadrática, ambos apresentaram $\mathrm{R}^{2}$ próximos, porém os agrupamentos que utilizaram a distância simples se apresentaram mais condizentes com a literatura. Já o método de Ward foi devido à sua característica de gerar grupos com poucos e aproximadamente o mesmo número de elementos, acarretando maior homogeneidade interna. Além disso, em comparação com os métodos do centroide e da ligação média, o método de Ward apresentou maior heterogeneidade entre os grupos, sendo esta obtida por meio do $\mathrm{R}^{2}$.

No agrupamento final, $70 \%$ da variabilidade de todo o agrupamento é explicada pela variabilidade entre os grupos, sendo os outros $30 \%$ explicados pela soma das variabilidades internas dos grupos (o $\mathrm{R}^{2}$ obtido foi de $70 \%$ ). O valor deste $\mathrm{R}^{2}$ encontra-se um pouco abaixo dos valores encontrados em Campos e Urraca Ruiz (2009) e em Campos (2005), porém os grupos gerados foram condizentes com a literatura econômica, na qual comumente são observados de quatro a seis clusters. O presente trabalho analisou alguns setores, como o de reciclagem, antes excluídos em outras pesquisas, porém mantidos nesta como forma de observar diferenças inter-setoriais específicas quanto ao processo de construção das inovações na economia brasileira.

É importante ressaltar que, para validação dos agrupamentos, foram observadas, sobretudo, questões teóricas, devido ao certo grau de subjetividade da análise de cluster, combinadas com as medidas de similaridade distintas, sendo escolhidos os agrupamentos obtidos pela combinação entre a distância euclidiana simples e o método de Ward, pelos motivos analisados anteriormente.

\section{RESULTADOS}

Por meio da análise de cluster especificada no item 3, observou-se inicialmente, através do dendrograma, a presença de sete ou três grupos. Para que fossem estabelecidos três clusters, os setores pertencentes ao grupo 3 e ao grupo 4 da Tabela 1 a seguir permaneceriam unidos, sendo isso não justificável do ponto de vista teórico. A separação em quatro grupos não foi justificável quando observados os gráficos em "teias" entre os grupos. Dessa forma, o agrupamento em seis clusters foi considerado o mais condizente com outras taxonomias, retratadas nas seções anteriores, obtendo-se uma heterogeneidade entre os grupos em torno de 70\%. A Tabela 1 a seguir mostra os agrupamentos finais: 
Tabela 1 - Agrupamentos setoriais derivados da análise de clusters

Grupo 1

\begin{tabular}{l}
\hline Fabricação de produtos alimentícios \\
\hline Fabricação de artigos de borracha e plástico \\
\hline Fabricação de produtos de minerais não metálicos \\
\hline Fabricação de produtos de metal \\
\hline Fabricação de produtos diversos \\
\hline Fabricação de produtos têxteis \\
\hline Confecção de artigos do vestuário e acessórios \\
\hline Preparação de couros e fabricação de artefatos de couro, artigos de viagem e calçados \\
\hline Fabricação de produtos de madeira \\
\hline Fabricação de papel, embalagens e artefatos de papel \\
\hline Edição, impressão e reprodução de gravações \\
\hline Metalurgia de metais não ferrosos e fundição \\
\hline Fabricação de artigos do mobiliário \\
\hline Fabricação e montagem de veículos automotores, reboques e carrocerias \\
\hline
\end{tabular}

\section{Grupo 3}

Fabricação de coque, álcool e elaboração de combustíveis nucleares

Refino de petróleo

\begin{tabular}{ll}
\hline \multicolumn{1}{c}{ Grupo 4 } \\
\hline Reciclagem & Grupo 5 \\
\hline Fabricação de bebidas & \\
\hline Fabricação de produtos do fumo & \\
\hline Fabricação de celulose e outras pastas & Produtos siderúrgicos \\
\hline Fabricação de máquinas para escritório e equipamentos de informática \\
\hline Fabricação de outros equipamentos de transporte
\end{tabular}

Fabricação de outros equipamentos de transporte

\section{Grupo 6}

Fabricação de produtos químicos

Fabricação de produtos farmacêuticos

Fabricação de máquinas e equipamentos

Fabricação de máquinas, aparelhos e materiais elétricos

Fabricação de material eletrônico básico

Fabricação de aparelhos e equipamentos de comunicações

Fabricação de equipamentos de instrumentação médico-hospitalares, instrumentos de precisão e ópticos, equipamentos para automação industrial, cronômetros e relógios 
Para analisar as semelhanças e diferenças entre os setores, têm-se a seguir os gráficos em "teias" para cada conjunto de variáveis, construídos através do vetor de médias para cada grupo, sendo estes calculados por meio da média ponderada pela participação da receita de determinado setor na receita total do cluster no qual ele se encontra inserido.

O Gráfico 1 consiste nas variáveis do primeiro conjunto, referente à importância atribuída à determinada atividade inovativa.

Nesse gráfico pode ser observado o comportamento homogêneo entre os grupos $1 \mathrm{e}$ 2 quanto à importância dada a essas variáveis. Por outro lado, os grupos 5 e 6 apresentam diferenças relevantes entre si e entre os demais grupos: o grupo 5 foi o que atribui maior importância ao treinamento e aos projetos industriais como atividade inovativa; por outro lado, o grupo 6 foi o que considerou a atividade de P\&D interno como de maior relevância. Uma relação comum a todos esses grupos foi o comportamento elevado, relativamente às outras variáveis, quanto à aquisição de máquinas e equipamentos (o grupo 6 foi o que apresentou índice menor, talvez devido à alta importância dada às atividades de $\mathrm{P} \& \mathrm{D}$ interno). Isso pode levar a supor que o processo de aprendizagem nos setores brasileiros está ocorrendo via conhecimentos externos implementados nessas máquinas e equipamentos, que, possivelmente, são importados.

\section{Gráfico 1 - Percentual de empresas que atribuíram alta importância aos itens indicados na teia}

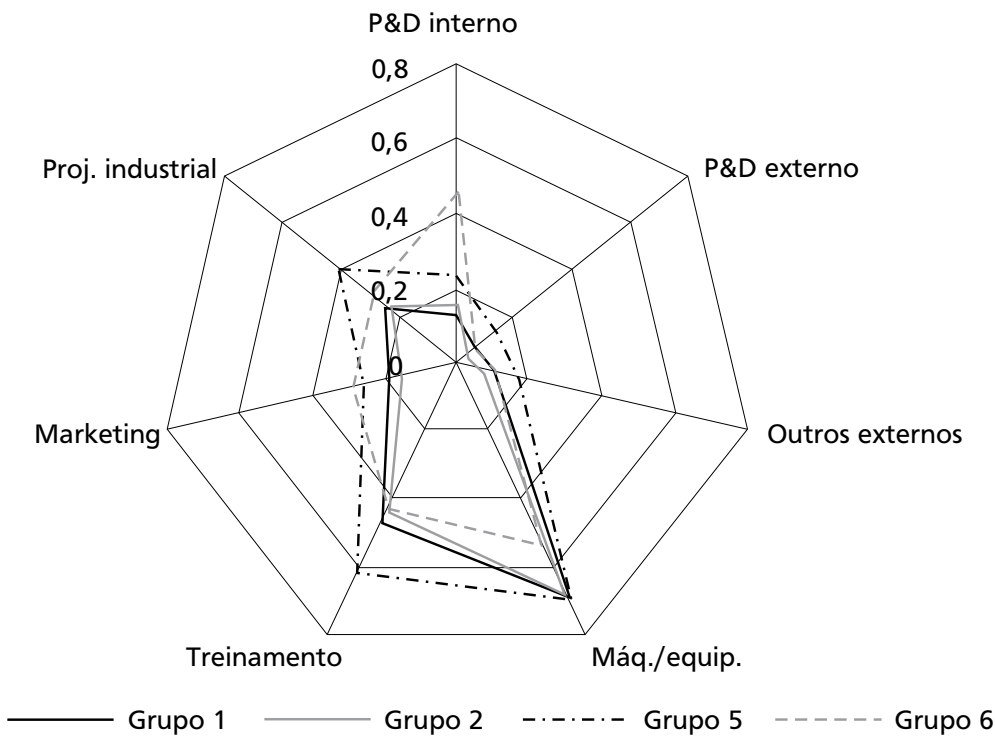


Gráfico 2 - Total de gastos em relação à receita líquida aplicados nos itens indicados na teia

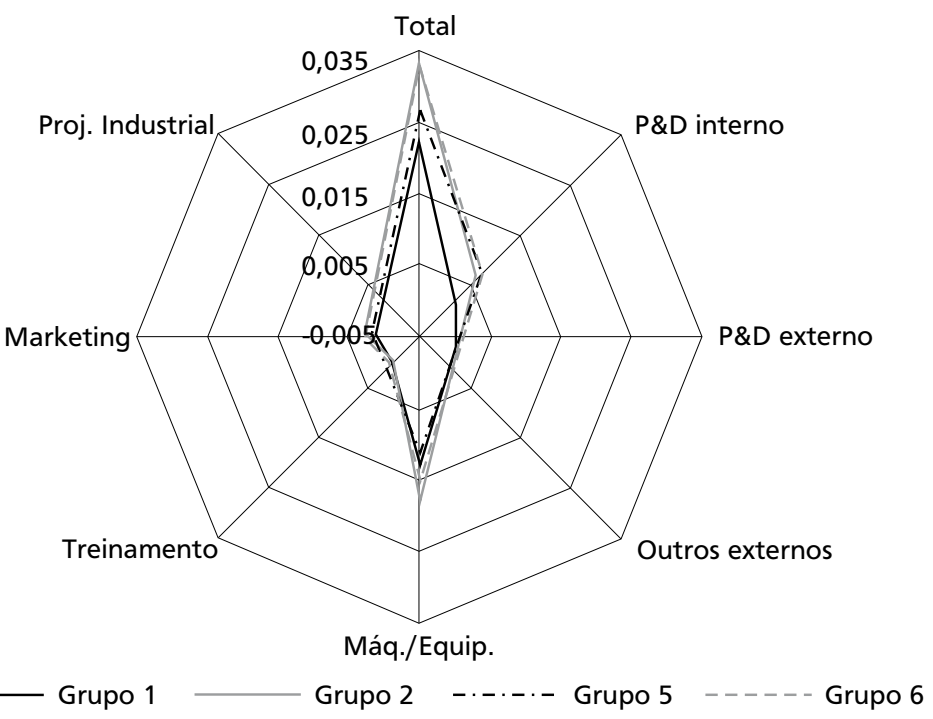

Fonte: Elaboração própria com base nos dados da PINTEC/IBGE.

No Gráfico 2 são enfatizados os esforços inovativos das empresas. Por esse gráfico, nota-se comportamento muito semelhante entre os setores, com pequena exceção para o primeiro grupo, o qual apresenta os menores índices tanto em relação ao total despendido quanto aos gastos com P\&D interno. É importante observar que o grupo 2 , mesmo tendo atribuído importância relativamente próxima à do grupo 1 para o primeiro conjunto de variáveis, realizou, em termos de suas receitas de vendas, gastos tão elevados quanto os dos grupos 5 e 6, os quais tinham atribuído maior relevância para as primeiras variáveis. É por meio desse gráfico que se observa umas das principais diferenças entre os grupos 1 e 2: a intensidade entre essas variáveis.

No Gráfico 3 está o gráfico referente ao caráter da atividade de P\&D e ao pessoal ocupado nessa atividade. Nele, observa-se novamente comportamento homogêneo entre os setores, com exceção do primeiro grupo, que obteve os maiores indicadores para o P\&D ocasional. Isso pode ser devido aos seus baixos índices para a atividade de $\mathrm{P} \& \mathrm{D}$ interno nos dois primeiros gráficos. 
Gráfico 3 - Total de gastos em relação à receita líquida aplicados em P\&D contínuo e ocasional e total relativo de pessoal com nível superior e dedicação exclusiva a P\&D

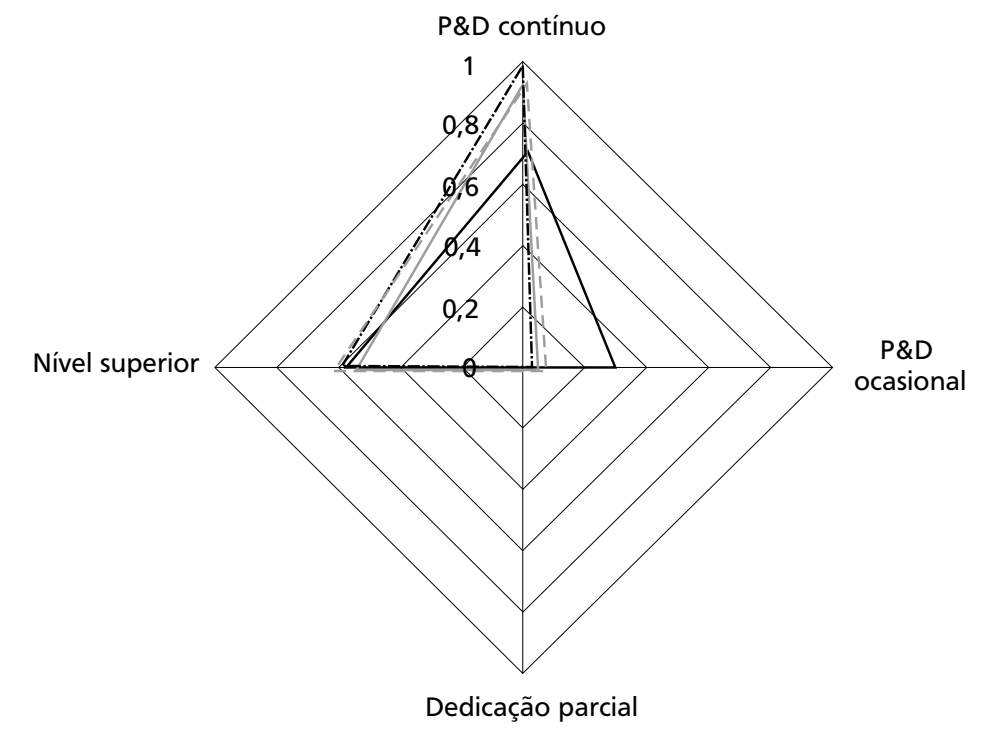

Grupo 1

Grupo 2 Grupo 5 Grupo 6

Fonte: Elaboração própria com base nos dados da PINTEC/IBGE.

Os Gráficos 4 e 5 indicam a importância atribuída à determinada fonte de conhecimento. Por meio do Gráfico 4, nota-se, novamente, comportamento semelhante entre os grupos 1 e 2, porém com diferença considerável quanto à utilização de fornecedores como fonte de informação, tendo o grupo 2 atribuído a maior importância para essa fonte. Isso vai ao encontro dos outros gráficos, em que o grupo 2 obteve indicadores relativamente elevados para a aquisição de máquinas e equipamentos. Novamente, para o grupo 6, têm-se os maiores indicadores quanto à utilização de departamentos internos de $\mathrm{P} \& \mathrm{D}$, corroborando as análises anteriores. Por outro lado, o grupo 5 foi o que apresentou maiores indicadores de importância para as outras áreas internas como fonte de informação, mostrando, como observado anteriormente, que ele não tem no P\&D interno sua principal forma de geração de inovações.

Através das outras fontes de conhecimento, representadas no Gráfico 5, nota-se a importância mais elevada dada pelo grupo 6 às redes informatizadas de informação; no que se refere à importância atribuída às universidades e centros de pesquisa como fonte de conhecimento, ele apresentou indicadores próximos aos dos grupos 5 e 1 . O grupo 2 foi o que obteve os menores indicadores quanto a essa variável, além de ter apresentado comportamento semelhante ao do grupo 1 nas demais variáveis. A importância 
atribuída às feiras e exposições foi praticamente a mesma para os grupos, com exceção do grupo 5, que atribui menor relevância, ao passo que ele conferiu, relativamente, importância um pouco maior quanto a patentes, licenças e know how.

Gráfico 4 - Percentual de empresas que atribuíram alta importância aos itens indicados na teia

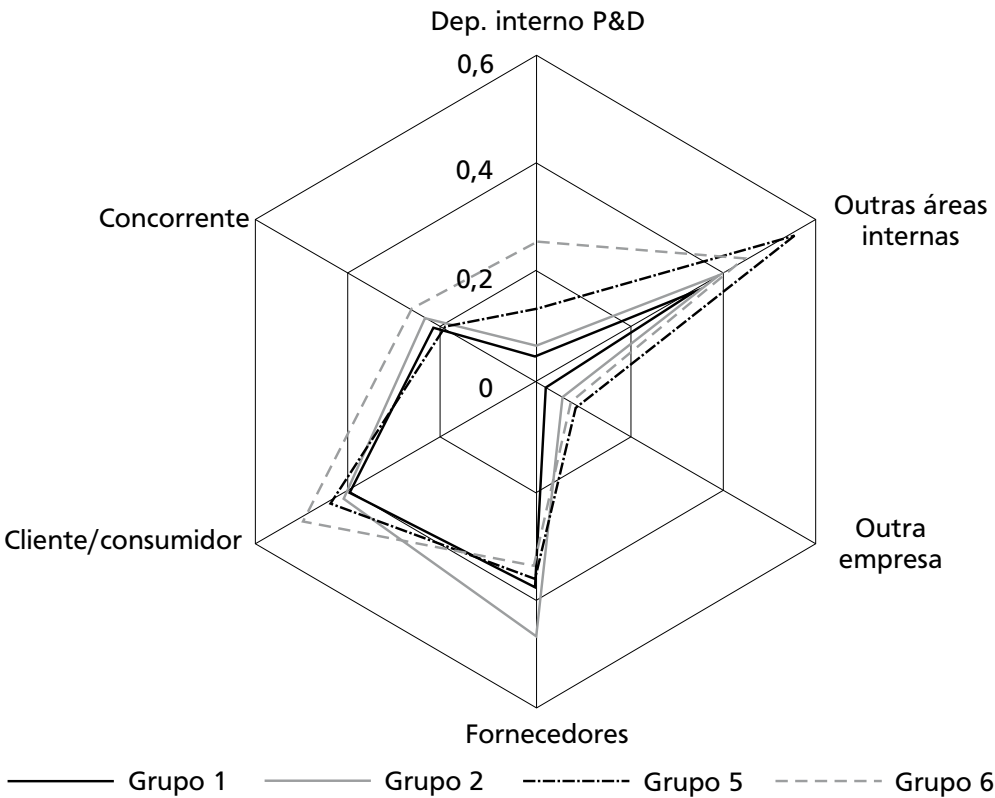

Fonte: Elaboração própria com base nos dados da PINTEC/IBGE. 


\section{Gráfico 5 - Percentual de empresas que atribuíram alta importância aos itens indicados na teia}

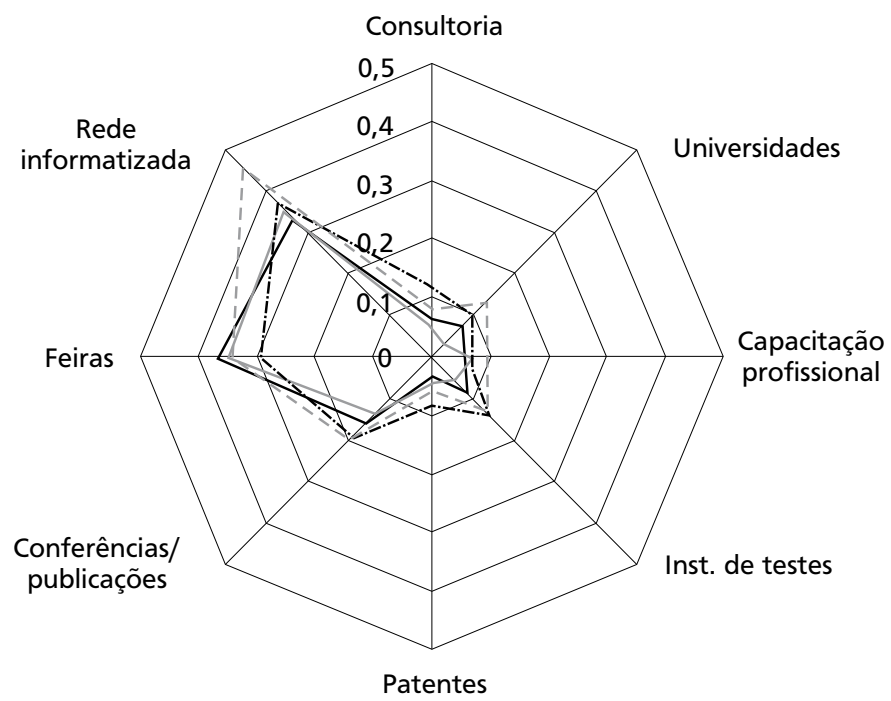

Grupo 1

Grupo 2

Grupo 5

Grupo 6

Fonte: Elaboração própria com base nos dados da PINTEC/IBGE.

Por meio do Gráfico 5 pode ser observada a maior discrepância entre os dois pares de grupos: os grupos 5 e 6 entre os 1 e 2. Os grupos 5 e 6 apresentam total de recursos despendidos muito mais elevado do que o dos grupos 1 e 2, além de apresentarem indicadores mais altos para praticamente todas as variáveis, com exceção da capacitação profissional. Uma observação relevante é a importância relativamente elevada dada pelo grupo 6 às cooperações com concorrentes, sendo esse comportamento não observado nos demais grupos. Isso pode ser um indício da complexidade das inovações geradas pelos setores desse grupo.

Com relação ao grupo 5, observa-se importância considerável para a relação de cooperação com clientes, consumidores e fornecedores, conforme mostrado no Gráfico 6. É interessante ressaltar que esse grupo não apresentou os maiores indicadores para esses mesmos agentes como fonte de conhecimento, sendo isso observado anteriormente. 
Gráfico 6 - Percentual de empresas que atribuíram alta importância aos itens indicados na teia Cliente/consumidor

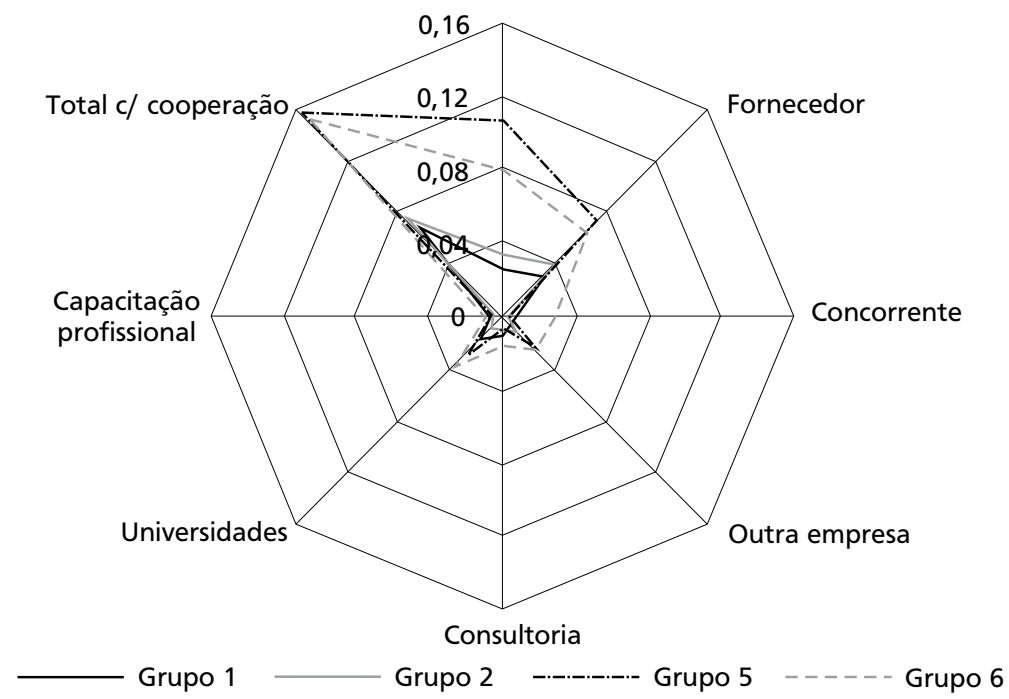

Fonte: Elaboração própria com base nos dados da PINTEC/IBGE.

Novamente, nota-se comportamento semelhante entre os grupos 1 e 2, com relativa diferença quanto à importância dada aos fornecedores, cliente e consumidores, além de o total gasto ser levemente diferente.

A observação exploratória dos gráficos mostra algumas diferenças importantes entre os grupos, como foi analisado. Contudo, pode-se supor que eles apresentam padrões, até certo ponto, semelhantes, diferenciando-se apenas quanto à intensidade em algumas variáveis. Isso pode revelar certa homogeneização do processo de aprendizagem e de geração de conhecimentos nos setores da economia brasileira. $O$ maior destaque é a aquisição de conhecimentos externos, como máquinas e equipamentos indicando uma tendência de homogeneização dos padrões de mudança tecnológica típicos de regimes tecnológicos caracterizados normalmente como de baixa tecnologia. Esse resultado demanda estudos mais aprofundados, mas é um resultado inédito em relação às taxonomias industriais propostas no contexto das economias desenvolvidas.

Caberia avaliar em que medida essa tendência de homogeneização do processo de aprendizagem está impactando as variáveis estruturais e de desempenho de cada grupo de setores. Idealmente, seria recomendável testar a qualidade dessa taxonomia analisando a dinâmica evolutiva de cada grupo setorial com ferramentas estatísticas mais elaboradas, a exemplo do que fez Marsili e Verspagen (2001). Nessa pesquisa optou-se por uma alternativa mais simples: a comparação com os agrupamentos de outras taxonomias, mais 
especificamente, a comparação com as taxonomias de Campos e Urraca Ruiz (2009) e de Marsili e Verspagen (2001), ambas apoiadas diretamente no conceito de regime tecnológico, sendo a primeira focada na economia brasileira e a segunda, nos testes de robustez.

Em relação à taxonomia de Campos e Urraca Ruiz (2009), foram realizados diversos processos de agrupamentos através de diferentes conjuntos de variáveis, sendo estas construídas a partir da PINTEC, referente ao período 1998-2000. Essas variáveis, sendo tanto inputs quanto outputs ao processo de inovação, encontram-se em cinco conjuntos: origem da inovação; tipos de conhecimento e sua procedência; foco da trajetória tecnológica; resultado da inovação; estrutura e desempenho (este último foi obtido pela Pesquisa Industrial Anual, também do IBGE, para o ano 2000).

Campos e Urraca Ruiz (2009) utilizaram apenas a análise de cluster para a determinação dos grupos. Esses autores fizeram uma análise de cluster para cada conjunto de variáveis, alocando, ao final, os setores segundo a taxonomia pavittiniana: dominados por fornecedores; fornecedores especializados; intensivos em economias de escala e de produção em massa; baseados na ciência e intensivos em P\&D.

Dos setores classificados por Campos e Urraca Ruiz (2009) como "dominados por fornecedores", a grande maioria foi classificado pelo presente trabalho nos grupos 1 e 2 da taxonomia final. Esses dois foram os que apresentaram os comportamentos mais próximos perante os conjuntos de variáveis analisadas, além de que foram os que mostraram os menores esforços inovativos, em termos de suas receitas líquidas, inclusive com as atividades de $\mathrm{P} \& \mathrm{D}$ interno, ao mesmo tempo em que se observou uma importância elevada para a aquisição de máquinas e equipamentos. Esses resultados são condizentes com os obtidos por Campos e Urraca Ruiz (2009), pois esses setores apresentaram, em tal trabalho, uma média importância para suas principais fontes de conhecimento, sendo estas em sua maioria fontes externas (principalmente aquisição de máquinas e equipamentos).

Em relação aos setores classificados como "fornecedores especializados", eles são compostos por alguns setores que estão nos grupos 5 e 6 da taxonomia final. Os dois setores que pertencem ao grupo 5 são "Fabricação de celulose e outras pastas" e "Fabricação de outros equipamentos de transporte", os quais, segundo Campos e Urraca Ruiz (2009) apresentaram formas de aprendizagem predominantes bem definidas (subcontratação e interação com universidades; e conhecimento tácito e pesquisa, respectivamente), enquanto os demais, que receberam a mesma classificação, apresentaram formas variadas. Isso pode ter sido relevante para a separação entre esses setores na taxonomia final, pois essa foi obtida utilizando apenas variáveis inputs ao processo de inovação, com destaque para as fontes de informação, inovação e cooperação.

A terceira classificação foi intitulada como "Intensivos em economias de escala e de produção em massa”. Enquanto nas classificações anteriores se observa um relativo predomínio de algum grupo da taxonomia final, nesta terceira classificação têm-se 
setores pertencentes aos grupos 5, 2 e 3 da taxonomia final. Nota-se em Campos e Urraca Ruiz (2009) que apenas a interação com universidades é uma forma comum de obtenção de conhecimento entre esses setores, porém cada um deles apresenta, até certo ponto, formas e fontes de conhecimento diferentes, o que explicaria a heterogeneidade entre essa classificação pavittiniana e a taxonomia final.

A última classificação indica alguns setores como "baseados na ciência e intensivos em P\&D”. Pode ser observado que a grande parte dos setores classificados como tal está no grupo 6 da taxonomia final. Por meio da análise gráfica, nota-se que esse grupo 6 foi o que apresentou as maiores importâncias para as fontes internas de $\mathrm{P} \& \mathrm{D}$, como o próprio departamento interno de $\mathrm{P} \& \mathrm{D}$, ao mesmo tempo em que atribuiu uma menor relevância para a aquisição de máquinas e equipamentos, sendo isso condizente com as observações realizadas por Campos e Urraca Ruiz (2009). Além disso, esses dois autores mostraram que não há um padrão específico quanto à forma de aprendizagem e à fonte de conhecimento. Isso pode ser confirmado no presente trabalho através dos gráficos em teia, nos quais esse grupo 6 apresentou indicadores relativamente elevados para diversas fontes de conhecimento, bem como para diferentes relações de cooperação.

Dessa forma, em especial, nota-se que este grupo 6 pode ser representado tanto por setores considerados como fornecedores especializados quanto por aqueles de maior potencial inovador (baseados em ciência e intensivos em P\&D). Isso pode fortalecer a suposição de que esteja ocorrendo certa homogeneização, a níveis considerados baixos, no processo de aprendizado e obtenção de conhecimento tecnológico na economia brasileira.

Nessa comparação é possível verificar que existe convergência de uma parte significativa dos setores. A maioria dos setores do grupo 1 e 2 foram classificados como "dominados por fornecedores" em Campos e Urraca Ruiz (2009) e quase todos os setores do grupo 6 foram classificados de "baseados em ciências" por esses autores.

A taxonomia proposta por Marsili e Verspagen (2001) foi construída com base em dados referentes às empresas holandesas para os anos entre 1994 e 1996. Esses dois autores buscam testar a tipologia de Marsili (2001), na qual são estabelecidos cinco regimes tecnológicos distintos: science-based (baseados em ciência); fundamental processes (processos básicos); complex systems (sistemas complexos); product engineering (engenharia de produto); continuous processes (processos contínuos).

Comparando o primeiro grupo da taxonomia final à análise de Marsili e Vespagem (2001), nota-se que grande parte dos setores tem seu regime tecnológico considerado como engenharia de produto (product engineering), no qual a principal fonte de conhecimento externo são os clientes/consumidores, ao mesmo tempo em que a relação de cooperação com estes é muito baixa. Ao observar a análise gráfica, nota-se que esse grupo não foi o que apresentou os maiores indicadores referentes a essa fonte; porém, foi uma das principais fontes de conhecimento utilizadas por ele. 
Esses autores ainda ressaltam que estes setores possuem os menores indicadores quanto às fontes de conhecimento, enquanto que os setores dos outros grupos apresentam padrões próximos. Isso poderia explicar o comportamento semelhante entre os setores, em relação a algumas variáveis referentes às fontes de informação.

Já o grupo 2 da taxonomia final é composto apenas por setores com regimes tecnológicos baseados em processos contínuos (continuous processes), nos quais é baixa a importância atribuída às fontes internas de $\mathrm{P} \& \mathrm{D}$, aos clientes/consumidores, aos concorrentes, e às universidades como fontes de conhecimento, enquanto a principal fonte são os fornecedores. A análise gráfica confirma a predominância dos fornecedores como fonte de conhecimento e a baixa utilização do departamento interno de P\&D para o mesmo fim, mas apresenta os concorrentes como uma fonte importante de informação.

É importante observar que o grupo 1 da taxonomia final também apresentou setores com regimes baseados na engenharia de produtos (product engineering). Isso pode ter ocorrido devido às semelhanças entre esse regime e o de continuous processes, como, por exemplo, a falta de cooperação com clientes e de investimentos internos em P\&D, além da pequena importância atribuída as universidades como fontes de conhecimento. Porém, esses dois regimes apresentam diferenças relevantes, como o próprio nível de oportunidade tecnológica, o que nos permite, utilizando também a análise gráfica como referência, supor que na economia brasileira os setores considerados como continuous processes, mas alocados no primeiro grupo da taxonomia final, apresentem padrões mais próximos do regime tecnológico product engineering.

Com relação ao grupo 5 da taxonomia final, esse foi, do ponto de vista teórico, o mais heterogêneo, apresentando setores classificados em todos os regimes, com exceção para o regime tecnológico de processos básicos (fundamental processes). Isso vai ao encontro das demais comparações realizadas anteriormente, como a comparação ao trabalho de Campos e Urraca Ruiz (2009). Isso pode nos mostrar, novamente, uma possível homogeneização no processo de inovação, a níveis baixos de esforços inovativos, pois foram agrupados tanto setores com média ou alta oportunidade tecnológica (como os de regimes complex systems e science-based), quanto setores com baixa oportunidade tecnológica. É importante ressaltar que o único setor deste quinto grupo considerado science-based foi o de fabricação de máquinas para escritório e equipamentos de informática, o que nos leva a supor que o mesmo esteja adquirindo conhecimento a partir da importação de máquinas e equipamentos.

Já o grupo 6 da taxonomia final apresentou tanto setores considerados por Marsili e Vespagem (2001) com alta e com média oportunidade tecnológica quanto, respectivamente, os regimes science-based e product engineering. Esses dois regimes se diferem basicamente pelos investimentos realizados em $\mathrm{P} \& \mathrm{D}$ interno, sendo que o primeiro atribui maior importância do que o segundo, ao mesmo tempo em que apresentam o 
mesmo grau de cumulatividade. Além disso, os setores baseados na ciência atribuem alta importância para as universidades e para a relação de cooperação com os clientes/ consumidores, se diferenciando do outro regime.

A junção do mesmo pode ter ocorrido devido à semelhança quanto ao grau de cumulatividade desses regimes. Juntamente a esse ponto, supõe-se que a junção tenha ocorrido devido a uma homogeneização do processo inovativo, onde, como suposto anteriormente, os setores que atribuem alta importância quanto aos investimentos internos em $\mathrm{P} \& \mathrm{D}$, aos níveis de esforços inovativos e às relações de cooperação, possam estar diminuindo seus esforços inovativos, se aproximando de setores onde esses esforços são menores.

Neste grupo 6, ainda há o setor de fabricação de produtos químicos, o qual foi considerado um regime tecnológico baseado em processos básicos, onde o grau de cumulatividade foi considerado alto por esses dos autores, se assemelhando aos demais setores considerados science-based. Além disso, esses setores também atribuem alta importância para as universidades como fonte de informação e para a relação de cooperação com os clientes/consumidores.

Com essa comparação, fica evidente a importância do contexto histórico na construção da taxonomia. O Brasil de 2000-2005 difere, obviamente, da Holanda de 19941996. Não obstante, a maior parte dos setores formou grupos semelhantes aos da taxonomia de Marsili e Verspagen (2001). E maior parte das diferenças nasceu da tendência de homogeneização do processo de aprendizagem dos setores da economia brasileira, detectada nesta pesquisa.

Um passo decisivo para o avanço desta pesquisa é a implementação de testes de robustez da taxonomia proposta, seguindo, por exemplo, o modelo adotado por Marsili e Verspagen (2001). Outra opção é estender o processo de comparação para verificar o grau de convergência incluindo outras taxonomias não diretamente vinculadas com o conceito de regime tecnológico. Acreditamos que essa via de teste é tão importante quanto os outros modelos. Essa via foi brevemente explorada nesta pesquisa, com resultados satisfatórios.

\section{CONCLUSÕES}

As características essenciais do processo de aprendizagem tecnológica - apropriabilidade, complexidade e oportunidades tecnológicas do conhecimento adquirido - definem o regime tecnológico prevalecente em cada setor. Neste artigo foi adotada a hipótese de que o regime tecnológico condiciona as decisões das firmas no que se refere à utilização e ao montante de recursos alocados para a atividade inovativa, definindo também as características essenciais das interações competitivas e não competitivas das firmas. Portanto, de acordo com essa hipótese, os padrões setoriais de inovação e de mudança tecnológica emergem do regime tecnológico prevalecente em cada setor. 
A tarefa de construir taxonomias embasadas no conceito de regime tecnológico está sendo posta em prática por autores da corrente neoschumpeteriana. Em alguns casos, técnicas estatísticas mais sofisticadas são usadas nessa construção, a exemplo da análise fatorial e de cluster. Há casos em que são empregadas técnicas de classificação menos formais, a exemplo da opinião de experts. Apesar das limitações inerentes a esse tipo de pesquisa, os resultados empíricos mostraram a relevância dessas taxonomias para os estudos dos padrões setoriais de mudança tecnológica e de competitividade externa.

Do ponto de vista metodológico, a taxonomia industrial apresentada nesta pesquisa é condizente com a linha de pesquisa que enfatiza as variáveis indicadoras do esforço inovativo das firmas como referência para classificação industrial. A escolha dessas variáveis deve-se à convicção de que elas são mais indicadas para a caracterização empírica do regime tecnológico prevalecente em cada setor.

O uso exclusivo da análise de cluster é outro aspecto importante da taxonomia apresentada neste artigo. Essa opção metodológica apresentou uma vantagem adicional no sentido de selecionar um conjunto de variáveis, cuja matriz de correlação indica não haver multicolinearidade entre essas variáveis.

Os resultados da análise de cluster apontam para o predomínio na indústria brasileira de quatro grupos setoriais característicos. Entretanto, a diferença entre esses grupos é sutil, pois, do conjunto de variáveis analisadas, são poucas as que geram essa diferença, não havendo desníveis profundos entre essas variáveis. Parece haver tendência de predomínio de setores com baixa intensidade em termos de esforços inovativos. A variável mais destacada em todos os setores como uma fonte importante de inovação é a aquisição de novas máquinas e equipamentos. Isso é típico de setores normalmente classificados como de baixa intensidade tecnológica em diversas taxonomias.

Não foram realizados testes de robustez desta taxonomia. Porém, fez uma breve comparação com algumas outras taxonomias. O grau de convergência foi relativamente alto, dando indícios de que a opção metodológica adotada nesta pesquisa é caminho promissor na identificação dos padrões de mudança tecnológica compatível com a realidade da economia brasileira.

\section{REFERÊNCIAS}

CAMPOS, B.; URRACA RUIZ, A. Padrões setoriais de inovação na indústria brasileira. Revista Brasileira de Inovação, v. 1, n. 8, p. 167-210, 2009.

COHEN, W.; LEVINTHAL, D. Innovation and learning: two faces of R\&D. The Economic Journal, v. 99 , p. 569-596, 1989. 
DOSI, G. Technological paradigms and technological trajectories: a suggested interpretation of the determinants and directions of technical change. Research Policy, v. 11, n. 3, p. 147-162, 1982.

DOSI, G. Sources, procedures and microeconomic effects of innovation. Journal of Economic Literature, n. 26, p. 1120-1171, set. 1988

GUIDOLIN, M. G.; MARTINELLI, O. M. Regimes tecnológicos da indústria brasileira: uma contribuição para a análise empírica. In: Encontro Nacional dos Centros Nacionais de PósGraduação em Economia - ANPEC, 36, Salvador, Dez. 9-12, 2008.

LALL, S. The technological structure and performance of developing country manufactured exports, 1985-1998. QEH Working Paper Series, n. 44, 2000.

MALERBA, F. Learning by firms and incremental technical change. Economic Journal, v. 102, p. 845-859, 1992.

MALERBA F.; ORSENIGO L. Technological regimes and firm behavior. Industrial and Corporate Change, v. 2, p. 45-74, 1993.

MALERBA F; ORSENIGO L. Technological regimes and sectoral patterns of innovative activities. Industrial and Corporate Change, v. 6, p. 83-117, 1997.

MARSILI, O. Technological regimes: theory and evidence. Working Paper, DYNACOM, 1999. Disponível em: <http://www.lem.sssup.it/Dynacom/files/D20_0.pdf>. Acesso em: 27 mar. 2012.

MARSILI, O. The anatomy and evolution of industries, technological change and industrial dynamics. Cheltenham: Edward Elgar, 2001.

MARSILI, O.; VERSPAGEN, B. Technological regimes and innovation: looking for regularities in Dutch manufacturing. Working Paper, ECIS, Eindhoven University of Technology, 2001.

NELSON, R.; WINTER, S. G. An evolutionary theory of economic change. Cambridge, Massachusetts: Harvard University, 1982.

ORGANIZAÇÃO PARA A COOPERAÇÃO E DESENVOLVIMENTO ECONÔMICO (OCDE). OECD Science, technology and industry scoreboard. Paris: OCDE, 2007

ORGANIZAÇÃO PARA COOPERAÇÃO E DESENVOLVIMENTO ECONÔMICO (OCDE). Manual de Oslo: Proposta de diretrizes para coleta e interpretação de dados sobre inovação tecnológica. FINEP/OCDE, 2004.

PAVITT, K. Sectoral patterns of technical change: towards a taxonomy and theory. Research Policy, n. 13, p. 343-373, 1984.

INSTITUTO BRASILEIRO DE GEOGRAFIA E ESTATÍSTICAS (IBGE). Pesquisa Nacional de Inovação Tecnológica - PINTEC. Rio de Janeiro: IBGE, 2005.

WINTER, S. Schumpeterian competition in alternative technological regimes. Journal of Economic Behavior and Organization, v. 5, p. 287-320, 1984.

UNITED NATIONS CONFERENCE ON TRADE AND DEVELOPMENT (UNCTAD). Trade and development report. Geneva: United Nations, 2002, p. 87-92. 


\section{ANEXO}

\section{Tabela A1 - Variáveis de caracterização setorial para análise de clusters}

Conjunto A - Total (\%) ${ }^{1}$ de empresas que atribuíram alta importância a:

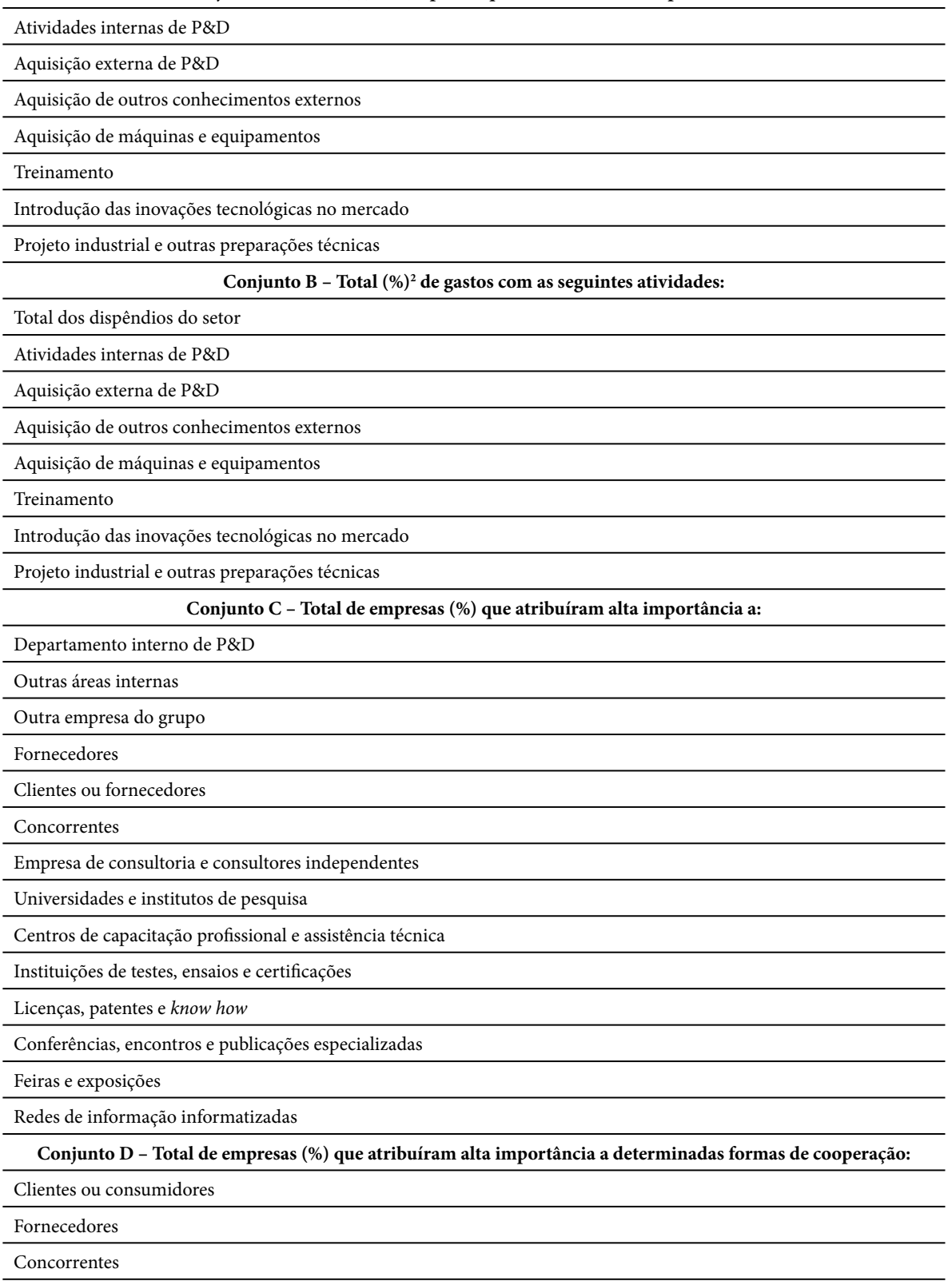




\begin{tabular}{l}
\hline Outra empresa do grupo \\
\hline Empresas de consultoria \\
\hline Universidades e institutos de pesquisa \\
\hline Centros de capacitação profissional e assistência técnica \\
\hline $\begin{array}{l}\text { Empresas que implementaram inovação, tendo relação de cooperação com outras organizações, em comparação ao } \\
\text { total dessas empresas }\end{array}$ \\
\hline Conjunto E - Total (\%) de gastos e de pessoal $(\%)^{3}$ com o seguinte caráter: \\
\hline Caráter contínuo das atividades internas de P\&D \\
\hline Pessoal em P\&D com dedicação parcial \\
\hline Pessoal nível superior ocupado em atividades internas de P\&D
\end{tabular}

Notas:

${ }^{1}$ Em relação ao total de empresas inovantes.

${ }^{2}$ Em relação à receita líquida total.

${ }^{3}$ Em relação ao total de empregados. 